\title{
On the uniqueness of solutions of the homogeneous curvature equations
}

by

\author{
Timothy R. CRANNY
}

Centre for Mathematics and its Applications

Australian National University, Canberra, ACT 0200, Australia

AbStRACT. - We prove that viscosity solutions of the degenerate prescribed curvature equation $F[u]=F\left(\kappa_{1}, \ldots, \kappa_{n}\right) \equiv 0$ in $\Omega, u=g$ on $\partial \Omega$ are unique for a broad class of differential operators $F$.

\section{INTRODUCTION}

We consider in this paper the fully nonlinear partial differential equation

$$
F[u]=F\left(\kappa_{1}, \ldots, \kappa_{n}\right)=0 \text { in } \Omega
$$

where $\Omega$ is a bounded domain in $\mathbb{R}^{n}$, and $F$ is a continuous function of the $\kappa_{i}$ 's, the principal curvatures of the graph of $u$. Since there is no intrinsic ordering of these curvatures, we restrict our attention to symmetric $F$. It is therefore natural to consider examples of functions $F$ such as the $k$ 'th-order elementary symmetric functions (for $1 \leq k \leq n$ )

$$
F(\kappa)=H_{k}(\kappa)=\sum \kappa_{i_{1}} \kappa_{i_{2}} \ldots \kappa_{i_{k}}
$$

where the summation is over all strictly increasing $k$-tuples $\left\{i_{1}, \ldots, i_{k}\right\} \subset$ $\{1,2, . ., n\}$. This family of functions includes the mean, scalar and Gauss 
curvatures by taking $k=1,2$ and $n$ respectively. By defining $H_{0}$ to be 1 , and considering quotients of the form

$$
F(\kappa)=H_{k, l}(\kappa)=\frac{H_{k}(\kappa)}{H_{l}(\kappa)} \quad \text { for } 0 \leq l<k \leq n,
$$

we include more general equations such as the harmonic curvature equation (corresponding to $k=n, l=n-1$ ). Such equations and the related Hessian equations have been used by Trudinger [6] to derive new isoperimetric inequalities. For the classical theory of such equations, see [2], [4] and references therein.

In the paper [5], Trudinger considered the problem

$$
\begin{aligned}
F[u]=F\left(\kappa_{1}, \ldots, \kappa_{n}\right) & =\psi & & \text { in } \Omega \\
u & =g & & \text { on } \partial \Omega
\end{aligned}
$$

with the functions $\psi$ and $g$ of insufficient smoothness to obtain classical solutions. It was therefore natural to consider the notion of viscosity solution introduced by Crandall and Lions [1]. This requires a little care, since equations of the form (1.1) are not elliptic for all functions $u$, but require the curvature vector $\kappa=\left(\kappa_{1}, . ., \kappa_{n}\right)$ of the graph of $u$ to lie in some suitable subset of $\mathbb{R}^{n}$, called the admissible set.

For the problems considered in [5], it is assumed that the admissible set is $\bar{K}$, where $K$ is an open symmetric cone in $\mathbb{R}^{n}$ with vertex at the origin and containing the positive cone. $F$ is assumed to satisfy the following:

$$
\begin{gathered}
F \in C^{0}(\bar{K}), \quad F>0 \text { in } K, \quad F=0 \text { on } \partial K \\
F(\kappa+\eta) \geq F(\kappa) \text { for all } \kappa \in K, \eta \geq 0 ; \\
F \text { is concave in } K ; \\
\sum F_{i} \geq \nu_{0}(F) \quad \text { in } K ; \\
\sum F_{i} \kappa_{i} \geq \nu_{1}(F) \quad \text { in } K ; \\
F_{i}(\kappa) \geq \nu_{2}(F) \quad \text { for any } \kappa \in K \text { with } \kappa_{i} \leq 0,
\end{gathered}
$$

where $\nu_{0}, \nu_{1}, \nu_{2}$ are positive nondecreasing functions on $\mathbb{R}^{+}$and the conditions involving the partial derivatives $F_{i}$ are taken to hold wherever the $F_{i}$ exist. 
To deal with merely continuous Dirichlet boundary data, interior gradient estimates are needed. To obtain these, a strengthened version of (1.10) is needed,

$$
F_{i}(\kappa) \geq \nu_{2}(F)+\nu_{3}(F) \sum F_{i} \quad \text { for any } \kappa \in K \text { with } \kappa_{i} \leq 0
$$

where $\nu_{3}$ is another positive nondecreasing function on $\mathbb{R}^{+}$.

To ensure the existence of barriers (also guaranteeing that the Dirichlet boundary condition holds in the strong sense), certain constraints are imposed on $\partial \Omega$. Letting $\kappa^{\prime}=\left(\kappa_{1}^{\prime}, \kappa_{2}^{\prime}, \ldots, \kappa_{n-1}^{\prime}\right)$ denote the principal curvatures of $\partial \Omega$ we require $\left(\kappa^{\prime}, 0\right) \in \bar{K}$, and

$$
\begin{gathered}
F\left(\kappa^{\prime}, 0\right) \geq \psi \quad \text { on } \partial \Omega ; \\
\lim \inf \frac{F(\kappa)-F\left(\kappa^{\prime}(y), 0\right)}{\kappa_{n}}>0 \quad \text { as } \quad \kappa_{n}+\frac{\left|\tilde{\kappa}-\kappa^{\prime}(y)\right|}{\kappa_{n}} \rightarrow 0,
\end{gathered}
$$

uniformly for $y \in \partial \Omega$, where $\tilde{\kappa}=\left(\kappa_{1}, . ., \kappa_{n-1}\right)$. Variations and refinements of the above conditions are discussed in [5].

The following theorem was proven in [5]:

THEOREM 1.1. - Let $F \in C^{0}(\bar{K})$ satisfy conditions (1.5) to (1.10), and let $\Omega$ be a bounded domain in $\mathbb{R}^{n}$ with $C^{2}$ boundary $\partial \Omega$ and $\psi \in C^{0,1}(\bar{\Omega})$ nonnegative satisfying (1.12), (1.13), $\nu_{0}(\inf \psi), \nu_{2}(\inf \psi)>0$. Then if there exists any bounded viscosity subsolution of (1.4) in $\Omega$, there exists for any $g \in C^{1,1}(\bar{\Omega})$ a viscosity solution $u \in C^{0,1}(\bar{\Omega})$ satisfying $u=g$ on $\partial \Omega$. If, in addition to the above structure conditions, (1.11) holds with $\nu_{3}(\inf \psi)>0$, then for any $g \in C^{0}(\partial \Omega)$ there exists a viscosity solution $u \in C^{0,1}(\Omega) \cap C^{0}(\bar{\Omega})$ satisfying $u=g$ on $\partial \Omega$.

Remark. - It should be noted that in [5] the conditions $\nu_{i}(\inf \psi)>0$ for $i=0,2,3$ are satisfied for the differential operators of primary interest even when inf $\psi=0$ by considering $F=\left(H_{k}\right)^{1 / k}$ and $\left(H_{k, l}\right)^{1 /(k-l)}$ rather than $H_{k}$ and $H_{k, l}$.

The principal curvatures $\kappa_{i}$ are the eigenvalues of the curvature matrix (second fundamental form) $\mathcal{H}$, which can be written as

$$
\mathcal{H}_{(p, r)}=\frac{1}{v} \mathcal{P} r \mathcal{P}
$$

where $v=\sqrt{1+|p|^{2}}, \mathcal{P}(p)=I-\frac{p \otimes p}{v(1+v)}$. It is then possible to think of the differential equation as $F[u]=\tilde{F}\left(\mathcal{H}_{\left(D u, D^{2} u\right)}\right)(x)=\psi(x)$ where $\tilde{F}$ is a function of the eigenvalues of the matrix-valued argument. The notion of 
admissible $\mathcal{H}$ is a trivial adaptation of that of admissible curvature vectors $\kappa \in \mathbb{R}^{n}$. We will at different times denote the differential operator by $F(\kappa)$, $F[u], F\left(D u, D^{2} u\right)$, or $\tilde{F}\left(\mathcal{H}_{\left(D u, D^{2} u\right)}\right)$.

The standard notion of a viscosity subsolution does not need modification to deal with prescribed curvature equations, but that of a supersolution needs to be altered since a function $\phi(\cdot)$ which touches $u$ from below at $x_{0}$ may satisfy $F[\phi]\left(x_{0}\right)>F[u]\left(x_{0}\right)$ if the curvatures of $\phi$ at $x_{0}$ lie outside the admissible set. The simplest example of this is $\phi(x)=A-B|x|^{2}$ for $B \gg 0$ and $F=H_{k}$ for $k$ even.

Following [5], we will say a function $u \in C^{0}(\Omega)$ is a viscosity subsolution of $F\left(D u(x), D^{2} u(x)\right)=\psi(x)$ if for any $x_{0} \in \Omega, \phi \in C^{2}(\Omega)$ satisfying $\phi \geq u$ in $\Omega, \phi\left(x_{0}\right)=u\left(x_{0}\right)$, one has $F\left(D \phi\left(x_{0}\right), D^{2} \phi\left(x_{0}\right)\right)-\psi\left(x_{0}\right) \geq 0$. However, we say $u \in C^{0}(\Omega)$ is a viscosity supersolution if for any $x_{0} \in \Omega, \phi \in C^{2}(\Omega)$ satisfying $\phi \leq u$ in $\Omega, \phi\left(x_{0}\right)=u\left(x_{0}\right)$, one has for $\eta \in \mathcal{S}^{n}$,

$$
\inf _{\eta \geq 0} F\left(D \phi\left(x_{0}\right), D^{2} \phi\left(x_{0}\right)+\eta\right)-\psi\left(x_{0}\right) \leq 0
$$

therefore avoiding the above-mentioned complications. It is also possible to define a supersolution by restricting one's attention to admissible smooth $\phi$.

The question of the uniqueness of solutions of (1.1) has remained an open question even for the case $\psi \geq \psi_{0}>0$ in $\Omega$ for some constant $\psi_{0}$. The standard techniques used in the theory of viscosity solutions are not applicable, since such curvature equations lack both the strict ellipticity and the strict monotonicity in the $z$ variable normally used to obtain uniqueness results. We show here that the above solution is unique in the highly degenerate case $\psi \equiv 0$. Subsequent work will consider the more general case of varying $\psi$.

The result proven here is:

THEOREM 1.2. - For $\Omega$ a bounded domain in $\mathbb{R}^{n}$, let $u \in C^{0}(\bar{\Omega})$ be a viscosity solution of the partial differential equation

$$
\begin{aligned}
F\left(D u, D^{2} u\right)=\tilde{F}\left(\mathcal{H}_{\left(D u, D^{2} u\right)}\right)(x) & =0 & & \text { in } \Omega \\
u & =g & & \text { on } \partial \Omega,
\end{aligned}
$$

where $F \in C^{0}\left(\mathbb{R}^{n} \times \mathcal{S}^{n}\right)$. If $F$ and $g$ are such that the above boundary condition always holds in the strong sense, and

$$
\tilde{F}(\mathcal{H}+c I)-\tilde{F}(\mathcal{H})>0
$$


holds for all admissible $\mathcal{H}$ and $c>0$, then the viscosity solution $u$ is unique in $C^{0}(\bar{\Omega})$.

The proof begins in a typical fashion by assuming that the solution $u$ is not unique, and considering the difference $u-v$ where $v$ is another solution. A geometric argument allows us to find a point in the interior of $\Omega$ where we have very good information about the difference in second derivatives, at the cost of some potentially awkward differences in the gradient terms. This point is typically not a point where $\sup (u-v)$ is attained, and its precise location is not determined by the arguments used.

In the following section we give a technical result which will allow us to exert some control over the location of this point, sufficient to show in the section that follows it that the useful information regarding the Hessians outweighs any possible problems introduced by gradient differences. We then obtain uniqueness results from a standard differential inequality argument.

\section{LOCALIZATION}

We show in this section that if $u$ and $v$ are 'reasonably nice' functions then the set upon which $\sup (u-v)$ is attained can be made arbitrarily small in diameter by making an arbitrarily small rigid-body perturbation of the graph of $u$. The result is intuitively reasonable, and it is likely that the proof below could be replaced with a more direct proof.

THEOREM 2.1. - If the functions $u$ and $v$ satisfy

1. $u$ and $-v$ are Lipschitz and semiconvex,

2. $\mathcal{C}=\left\{x \in \bar{\Omega} \mid(u-v)(x)=\sup _{\Omega}(u-v)\right\} \subset \subset \Omega$

then for any $\delta>0$ one may perform an arbitrarily small 'rigid-body' perturbation of the graph of u to produce a new Lipschitz and semiconvex function $\hat{u}$ such that $\hat{\mathcal{C}}=\left\{x \in \bar{\Omega} \mid(\hat{u}-v)(x)=\sup _{\Omega}(\hat{u}-v)\right\} \subset \subset B\left(x_{0}, \delta\right) \subset \subset \Omega$ for some point $x_{0} \in \Omega$.

Remark. - 1. The assumptions above on $u$ and $v$ will hold automatically in our applications since we will apply this result only to regularisations of our solutions. 2. By a 'rigid-body perturbation' of a function or its graph we mean changes corresponding to translations and rotations of the graph in $\mathbb{R}^{n+1}$. The magnitude of the perturbation can be measured in terms of greatest distance moved by any point on the graph. 3. One effect of any such perturbation is to slightly change the domain, but since our arguments 
are all concerned with points which are bounded away from $\partial \Omega$ and we only use arbitrarily small perturbations, we will ignore this effect.

The above result is a consequence of the following Theorem.

TheOREM 2.2. - Let $u, v$ and $\mathcal{C}$ be as in Theorem 2.1. For any $\bar{x} \in \mathcal{C}$ one may make an arbitrarily small rigid-body perturbation $\hat{u}$ of $u$ so as to ensure that

$$
B\left(\bar{x}, K_{0}\right) \cap \hat{\mathcal{C}}=\emptyset
$$

where

$$
K_{0}=C_{0} \min \left\{\frac{\operatorname{diam} \mathcal{C}}{2}, C_{1}\right\},
$$

for $C_{0}, C_{1} \in(0, \infty)$ constants depending only upon the Lipschitz and semiconvexity bounds on $u$ and $-v$.

Proof. - For the sake of simplicity let us temporarily shift $u$ vertically to consider the case where $\sup _{\Omega}(u-v)=0$. The idea behind the proof is to leave $v$ as it is, but to lower the graph of $u$ by $\tau<<1$, taking the two graphs out of contact. We then tilt the graph of $u-\tau$ in an appropriate direction until contact is re-established. We are able show that contact must be established for a certain small tilt angle, and that information tells us that the contact point cannot be too close to $\bar{x}$, since the small tilt cannot compensate for the drop in height until one is sufficiently far away from the 'axis' of rotation. (When talking of rotations we will use some of the language of $\mathbb{R}^{3}$, although all work is done in $\mathbb{R}^{n}$ for $n$ arbitrary.)

The only problem in using a rotation of the graph of $u-\tau$ to force renewal of the contact between the two graphs is that while one can easily make a point $(x, u(x)-\tau)$ trace out a portion of a vertical circle (and thereby rise as desired), it is conceivable that near the point in question the graph of $v(\cdot)$ has the same partially spherical shape, meaning that the improvement due to the tilting of $g r(u-\tau)$ brings $(x, u(x)-\tau)$ no closer to $g r v$. This problem can be ruled out by using semiconcavity and almost-everywhere differentiability and restricting one's attention to points which are sufficiently close together (this last point is responsible for the $C_{1}$ term in (2.2)).

Let us begin by choosing $\tau$ very small and fixed. Let $C$ be a constant such that

$$
u+C|x|^{2}, \quad-v+C|x|^{2} \quad \text { are convex }
$$


Since $u$ and $v$ are only twice-differentiable almost-everywhere, we use the now-standard arguments of Jensen to find a point $x_{0}$ extremely close to $\mathcal{C}$ such that

1. $u$ and $v$ are twice differentiable at $x_{0}$,

2. $(u-v)\left(x_{0}\right) \geq-\tau^{2}$,

3. $\left|D(u-v)\left(x_{0}\right)\right| \leq \tau^{2}$.

By (2.3) we have

$$
\begin{aligned}
& u\left(x_{0}\right)+D u\left(x_{0}\right) \cdot\left(x-x_{0}\right)-C\left|x-x_{0}\right|^{2} \\
& \quad \leq u(x) \leq v(x) \leq v\left(x_{0}\right)+D v\left(x_{0}\right) \cdot\left(x-x_{0}\right)+C\left|x-x_{0}\right|^{2}
\end{aligned}
$$

Since $u \leq v$ and $u$ is Lipschitz, it follows that

$$
\operatorname{dist}\{g r(u-\tau), g r v\} \geq \frac{\tau}{C_{n+1}},
$$

for some constant $C_{n+1}<\infty$, where $\operatorname{dist}\{A, B\}=$ $\inf \{|x-y| \mid x \in A, y \in B\}$ for $A, B \subset \mathbb{R}^{n+1}$.

We consider a point on $g r(u-\tau)$ sufficiently close to $\left(x_{0}, u\left(x_{0}\right)-\tau\right)$ and call it the origin in $\mathbb{R}^{n+1}$, (thereby ensuring that $\left|x_{0}\right|$ is sufficiently small). We choose our coordinate system for $\mathbb{R}^{n}$ so that $x_{0}$ lies along the $x_{n}$-axis, and tilt the graph of $u-\tau$ by angle $\sigma$ about the origin 'in the $x_{n}$ direction' by applying the linear operator $A_{\sigma}$ where the $(n+1) \times(n+1)$ matrix of $A_{\sigma}$ is given by

$$
A_{\sigma}=\left[\begin{array}{ccc|cc} 
& & & 0 & 0 \\
& I_{n-1} & & \vdots & \vdots \\
& & & 0 & 0 \\
\hline 0 & \cdots & 0 & \cos \sigma & -\sin \sigma \\
0 & \cdots & 0 & \sin \sigma & \cos \sigma
\end{array}\right]
$$

so under the action of $A_{\sigma}$ upon $g r(u-\tau), x_{1}, \ldots, x_{n-1}$ are unchanged, but $x_{n} \rightarrow \cos \sigma x_{n}-\sin \sigma(u(x)-\tau)$ and $(u(x)-\tau) \rightarrow \sin \sigma x_{n}+$ $\cos \sigma(u(x)-\tau)$. 
The distance a point $(x, u(x)-\tau)$ is moved by $A_{\sigma}$ is bounded (for $\sigma<<1$ ) by $2 \sigma C_{u}|x|$ where $C_{u}$ is the Lipschitz bound on $u$, so contact between the graphs cannot be re-established if

$$
|x| \leq \frac{\tau}{\sigma}\left(2 C_{u} C_{n+1}\right)^{-1}, \quad \text { for } \sigma \ll 1 .
$$

We now show that a tilting by angle $\sigma \leq C \tau\left|x_{0}\right|^{-1}$ (for some $C<\infty$ ) ensures contact between the two graphs, by showing that a tilting by angle $C \tau\left|x_{0}\right|^{-1}$ means that the point $\left(x_{0}, u\left(x_{0}\right)-\tau\right)$ is rotated to a point lying above the graph of $v$. Accordingly there must be some smaller angle for which contact first occurs (we do not know where or for what tilt angle this contact occurs).

We now concentrate on the two-dimensional vertical plane spanned by $x_{n}$ and $x_{n+1}$. This allows us to treat this aspect of the proceedings as a 1-dimensional problem.

Using (2.4) one can show by simple calculations that if $\left|x_{0}\right|$ is less than some constant $C_{1}$, then the path swept out by $\left(x_{0}, u\left(x_{0}\right)-\tau\right)$ upon tilting is almost perpendicular to the slope of $g r v$ at $x_{0}$. This means that as one tilts about the origin, $\left(x_{0}, u\left(x_{0}\right)-\tau\right)$ does not 'follow' the graph of $v$ at all, but instead lifts upwards (for the correct sign of $\sigma$ !) towards $g r v$. One can then easily show that for a tilt angle of $\sigma$ given by $\sigma\left|x_{0}\right|=C \tau$, (for some constant $C$ depending only on the Lipschitz and semiconcavity bounds for $u$ and $v$ ), the point $\left(x_{0}, u\left(x_{0}\right)-\tau\right)$ is swept above the graph of $v$. Clearly, for some smaller $\sigma$, contact between the two graphs is made for the first time, so for this $\sigma$ we have $\tau / \sigma \geq\left|x_{0}\right| C^{-1}$. Harking back to (2.7), we see that contact has taken place, but that the contact cannot be for $x$ such that

$$
|x| \leq\left|x_{0}\right|\left(2 C_{u} C C_{n+1}\right)^{-1} .
$$

Since we have the restriction that the origin and $x_{0}$ be within distance $C_{1}$ of each other, and we wish to consider the case $0 \in \mathcal{C}$ and also $x_{0}$ very close to $\mathcal{C}$, the right-hand side of the above expression can be written as $K_{0}=C_{0} \min \left\{\frac{\operatorname{diam} \mathcal{C}}{2}, C_{1}\right\}$. By relabeling our origin, we have the desired result.

The ball $B\left(0, K_{0}\right) \subset \mathbb{R}^{n}$ has therefore been 'bitten out' from the set upon which the new supremum is achieved. The important feature of the above result is that the size of the ball removed from contention depends only on the relative sizes of $\tau$ and $\sigma$, so one may trivially make the perturbation used as small as desired without changing the result in any way.

Remark. - It should be noted that we are not claiming that the new supremum set $\hat{\mathcal{C}}$ is $\mathcal{C} \backslash B\left(0, K_{0}\right)$ ! The less important reason is that the 
perturbation may bring points (barely) outside $\mathcal{C}$ into contention (one can easily control this tendency by decreasing the magnitude of the perturbation), while more significantly, what would typically happen is that the perturbation would produce a much smaller $\hat{\mathcal{C}}$, with the explicit removal of the ball above dramatically understating the 'pruning' done. For example, if $u$ and $v$ are affine a single perturbation can result in $\hat{\mathcal{C}}=\{\bar{x}\}$ for some $\bar{x} \in \Omega$. This example illustrates the principle that if one has any additional information the results used here can be replaced with a satisfyingly concrete construction, but unfortunately none are readily apparent in the more general case.

Proof of Theorem 2.1. - The result now follows easily from the repeated application of Theorem 2.2, due to the compactness of $\mathcal{C}$ and all subsequent versions thereof. The only aspect deserving of caution is the fact that each perturbation may allow points outside the previous supremum set to be brought into play. This can be easily avoided by using a simple compactness argument to add at each iteration the restriction that the perturbation be sufficiently small to ensure that far more is removed from contention than is introduced. For any given $\delta>0$ only a finite number of iterations is required to cut the supremum set down until it has diameter less than $\delta$. If one wishes the total perturbation to be at most of size $\tau_{0}$, then Theorem 2.2 is used with successive perturbations of at most size $\tau_{0} 2^{-i-1}$ for $i=1,2, \ldots$

\section{PROOF OF UNIQUENESS}

Proof of Theorem 1.2. - If $u$ is not unique, then there exists a second distinct viscosity solution $v \in C^{0}(\bar{\Omega})$. Since the boundary condition holds in the strong sense, $u(\cdot)=v(\cdot)=g(\cdot)$ on $\partial \Omega$ and without loss of generality we assume $M_{0}=\sup (u-v)>0$ and $\mathcal{C} \subset \subset \Omega$ for $\mathcal{C}=\left\{y \in \bar{\Omega} \mid(u-v)(y)=M_{0}\right\}$.

To avoid the problems caused by the lack of smoothness of $u$ and $v$, we take the standard route of considering the sup- and inf-convolutions $u_{\epsilon}^{+}, v_{\epsilon}^{-}$, first used in this context in [3] and defined by

$$
\begin{aligned}
& u_{\epsilon}^{+}(x)=\sup _{y \in \bar{\Omega}}\left\{u(y)-\frac{|x-y|^{2}}{\epsilon^{2}}\right\}, \\
& v_{\epsilon}^{-}(x)=\inf _{y \in \bar{\Omega}}\left\{v(y)+\frac{|x-y|^{2}}{\epsilon^{2}}\right\},
\end{aligned}
$$

Vol. 13, $\mathrm{n}^{\circ}$ 5-1996. 
for $\epsilon>0$. As shown in [3], $u_{\epsilon}^{+}$and $v_{\epsilon}^{-}$are also viscosity subsolution and supersolution respectively of (1.1) with the domain now slightly restricted (we use here the fact that the homogeneous curvature equation is independent of $x$ ).

The two functions are twice differentiable almost everywhere, with

$$
\begin{gathered}
\left|D u_{\epsilon}^{+}\right|,\left|D v_{\epsilon}^{-}\right| \leq \epsilon^{-1} \\
-2 \epsilon^{-2} I \leq D^{2} u_{\epsilon}^{+}, \quad 2 \epsilon^{-2} I \geq D^{2} v_{\epsilon}^{-}
\end{gathered}
$$

holding in the sense of distributions. It is clear that for $\epsilon$ sufficiently small, $u_{\epsilon}^{+}-v_{\epsilon}^{-}$will attain its supremum inside some set strictly contained in $\Omega$. We fix $\epsilon$ to be such a small constant. We now use the results of the previous section to perform a (small) rigid body perturbation of $u_{\epsilon}^{+}$so as to make the set upon which the supremum of $\hat{u_{\epsilon}^{+}}-v_{\epsilon}^{-}$is attained have diameter less than $\delta$ where $\delta$ is some constant sufficiently small with respect to $\epsilon$. This rigid-body perturbation does not change the principal curvatures of the graph, but does introduce some small change in where these curvatures are recorded. Since in this instance we are interested in the case where there is no spatial dependence, this makes no difference. This means that $\hat{u_{\epsilon}^{+}}$is a subsolution of the original equation, and $v_{\epsilon}^{-}$is a supersolution. For ease of notation we will refer to these functions as $\hat{u}$ and $v$.

We now take the origin in $\mathbb{R}^{n}$ to be the centre of the small ball containing the supremum set of $\hat{u}-v$. Consider the sphere $S$ in $\mathbb{R}^{n+1}$ with centre $(0,-A)$ and radius $R$, with $A(R)$ chosen so that $S$ lies above the graph of $\hat{u}-v$ and touches it at some point $\bar{x}$. By standard negligible tilting we may assume that the functions $\hat{u}$ and $v$ are twice differentiable at $\bar{x}$. We will derive a contradiction by evaluating the differential inequalities for $\hat{u}$ and $v$ at $\bar{x}$.

It is clear that:

$$
\begin{aligned}
D \hat{u}(\bar{x})-D v(\bar{x}) & =-\bar{x}\left(R^{2}-|\bar{x}|^{2}\right)^{-1 / 2} \\
D^{2} \hat{u}(\bar{x})-D^{2} v(\bar{x}) & \leq-\left(R^{2}-|\bar{x}|^{2}\right)^{-1 / 2}\left[\mathrm{I}+\frac{\bar{x} \otimes \bar{x}}{R^{2}-\bar{x}^{2}}\right] .
\end{aligned}
$$

The inequality for second derivatives gives us a very valuable tool in attempting to derive a differential inequality, but the difference in gradient terms is a complicating factor and one which can, to some extent, cancel out the benefits from the second derivative information. The reason for the localisation arguments in the previous section is that since the supremum of $\hat{u}-v$ is taken only in $B(0, \delta)$, it is clear that for $R$ sufficiently large, $|\bar{x}|<2 \delta$. This has precisely the effect of ensuring that the 'bad' gradient 
difference is much smaller than the 'good' Hessian difference term, since the gradient difference is $O\left(|\bar{x}| R^{-1}\right)$ for $R>>1$, while the Hessian difference is $O\left(R^{-1}\right)$. By choosing $\delta$ sufficiently small and then $R$ sufficiently large, we may make this imbalance as great as we wish.

We are now in a position to prove a differential inequality which contradicts (1.15) and proves the nonexistence of a second solution. One may proceed by using the information contained in (3.3) directly in (1.14) (a messy procedure!), but we shall illustrate further the utility of the rigidbody perturbation invariance of solutions by concluding the proof using that property.

One can tilt the graph of $\hat{u}$ about $(\bar{x}, \hat{u}(\bar{x}))$ so as to remove completely the difference between the gradients of $\hat{u}$ and $v$ at $\bar{x}$. Straightforward but tedious calculation shows that the magnitude of the change in $D_{i j} \hat{u}(\bar{x})$ caused by this tilting is bounded by some constant times the magnitude of the change in the gradient (i.e. $O\left(|\bar{x}| R^{-1}\right)$ ), with the constant factor controlling the change in the Hessian depending only upon $\epsilon$ (through the bounds on $D \hat{u}(\bar{x})$ and $D^{2} \hat{u}(\bar{x})$ ). Since (3.3) holds, and we may make $|\bar{x}|$ as small as we like by taking $\delta$ sufficently small and $R$ sufficiently large, after tilting we have a new function $u^{*}$ which is a subsolution of our original equation and satisfies

$$
\begin{aligned}
D u^{*}(\bar{x}) & =D v(\bar{x}) \\
D^{2} u^{*}(\bar{x}) & \leq D^{2} v(\bar{x})-\frac{1}{2}\left(R^{2}-|\bar{x}|^{2}\right)^{-1 / 2}\left[\mathrm{I}+\frac{\bar{x} \otimes \bar{x}}{R^{2}-\bar{x}^{2}}\right] .
\end{aligned}
$$

We then have at $\bar{x}$,

$$
\mathcal{H}_{\left(D v, D^{2} v\right)} \geq \mathcal{H}_{\left(D u^{*}, D^{2} u^{*}+(3 R)^{-1} I\right)} \geq \mathcal{H}_{\left(D u^{*}, D^{2} u^{*}\right)}+c \mathrm{I}
$$

where $c>0$ is some constant depending only on $\epsilon$ and $R$ (this follows directly from the definition of $\mathcal{H}$ ). It follows that at $\bar{x}$,

So we have

$$
\begin{aligned}
F\left(D v, D^{2} v\right) & =\tilde{F}\left(\mathcal{H}_{\left(D v, D^{2} v\right)}\right) \\
& \geq \tilde{F}\left(\mathcal{H}_{\left(D u^{*}, D^{2} u^{*}\right)}+c \mathrm{I}\right) \\
& >\tilde{F}\left(\mathcal{H}_{\left(D u^{*}, D^{2} u^{*}\right)}\right) \geq 0
\end{aligned}
$$

$$
F\left(D v(\bar{x}), D^{2} v(\bar{x})\right) \geq a>0,
$$

with the same true if we replace $D^{2} v$ with $D^{2} v+\eta$ for $\eta \geq 0$ in $\mathcal{S}^{n}$, since $D^{2} v \geq D^{2} \hat{u}$, so there exists $\phi \in C^{2}(\Omega)$ touching $v$ from below at some point $y$ such that

$$
\inf _{\eta \geq 0} F\left(D \phi(y), D^{2} \phi(y)+\eta\right)>0,
$$

in contradiction of (1.15). 


\section{CONCLUDING REMARKS}

The techniques used here have relied on several occasions upon the fact we are dealing with the homogeneous prescribed curvature problem rather than the more general $F\left(\kappa_{1}, \kappa_{2}, \ldots, \kappa_{n}\right)=\psi(x) \geq 0$. The use of the above techniques in this more general context is the focus of work in progress. As is typical in viscosity theory, the spatial dependence in the inhomogeneous case introduces complications due to the effect of the regularisation process. It is interesting to note that the rigid-body perturbation process is similarly affected, but the spatial shifting there is somewhat amenable to control. It is possible that the perturbation process can be actively used to mitigate the effect of the regularisation process.

\section{ACKNOWLEDGEMENTS}

The author would like to express his deep appreciation for the help of Drs John Urbas and Alex Isaev and Professors Neil Trudinger and Nina Ivochkina in a number of very useful discussions during the development of this work.

\section{REFERENCES}

[1] M. G. Crandall and P.-L. Lions, Viscosity solutions of Hamilton-Jacobi equations, Trans. Amer. Math. Soc., Vol. 277, 1983, pp. 1-42.

[2] N. N. IvochkinA, Solution of the Dirichlet problem for curvature equations of order $m$, Mat. Sb., Vol. 180, 1989, pp. 867-887; English trans. Math. USSR. Sb., Vol. 67, 1990, pp. 317-339.

[3] R. Jensen, P.-L. Lions and P. E. Souganidis, A uniqueness result for viscosity solutions of second order fully nonlinear partial differential equations, Proc. Amer. Math. Soc., Vol. 102, 1988, pp. 975-978.

[4] M. LiN and N. S. TRUdingeR, The Dirichlet problem for the prescribed curvature quotient equations, Top. Methods in Nonlinear Analysis, Vol. 3, 1994, pp. 1-17.

[5] N. S. Trudinger, The Dirichlet problem for the prescribed curvature equations, Arch. Rat. Mech. Anal., Vol. 111, 1990, pp. 153-179.

[6] N. S. Trudinger, Isoperimetric inequalities for quermassintegrals, Ann. l'Inst. Henri Poincaré. Analyse non linéaire, Vol. 11 (4), 1994, pp. 411-425. 